\title{
A game-theoretic analysis of the implications of overlay network traffic on ISP peering
}

\author{
Jessie Hui Wang ${ }^{a}$, Dah Ming Chiu ${ }^{b, *}$, John C.S. Lui ${ }^{c}$ \\ a Network Research Center, Tsinghua University, Tsinghua National Laboratory of Information Science and Technology, China \\ ${ }^{\mathrm{b}}$ Department of Information Engineering, The Chinese University of Hong Kong, Room 814, HSH Building, Shatin, NT, Hong Kong \\ ${ }^{\mathrm{c}}$ Department of Computer Science and Engineering, The Chinese University of Hong Kong, Hong Kong
}

\section{A R T I C L E I N F O}

\section{Article history:}

Received 30 April 2007

Received in revised form 23 June 2008

Accepted 24 June 2008

Available online 1 July 2008

Responsible Editor U. Krieger

\section{Keywords:}

ISP

Peering

Traffic model

Game theory

Network management

\begin{abstract}
A B S T R A C T
Inter-ISP traffic flow determines the settlement between ISPs and affects the perceived performance of ISP services. In today's Internet, the inter-ISP traffic flow patterns are controlled not only by ISPs' policy-based routing configuration and traffic engineering, but also by application layer routing. The goal of this paper is to study the economic implications of this shift in Internet traffic control assuming rational ISPs and subscribers. For this purpose, we build a general traffic model that predicts traffic patterns based on subscriber distribution and abstract traffic controls such as caching functions and performance sensitivity functions. We also build a game-theoretic model of subscribers picking ISPs, and ISPs making provisioning and peering decisions. In particular, we apply this to a local market where two ISPs compete for market share of subscribers under two traffic patterns: "Web" and "P2P overlay", that typifies the transition the current Internet is going through. Our methodology can be used to quantitatively demonstrate that (1) while economy of scale is the predominant property of the competitive ISP market, P2P traffic may introduce unfair distribution of peering benefit (i.e. free-riding); (2) the large ISP can restore more fairness by reducing its private capacity (bandwidth throttling), which has the drawback of hurting business growth; and (3) ISPs can reduce the level of peering (e.g. by reducing peering bandwidth) to restore more fairness, but this has the side-effect of also reducing the ISPs' collective bargaining power towards subscribers.
\end{abstract}

(c) 2008 Elsevier B.V. All rights reserved.

\section{Introduction}

The Internet is operated by many internet service providers (ISPs) who decide to interconnect their networks together. There are primarily two kinds of peering relationships between Internet ISPs: (i) provider to customer relationship and (ii) free peering (also called peer to peer) relationship [7]. These peering relationships define a set of transit service agreements [11-13]. For example, under the provider to customer relationship, the customer ISP pays the provider ISP for the traffic exchange on the peering link between them; while the traffic exchange on the

\footnotetext{
* Corresponding author. Tel.: +852 2609 8357; fax: +852 26035032.

E-mail addresses: hwang@cernet.edu.cn (J.H. Wang), dmchiu@ie.cuhk. edu.hk (D.M. Chiu), cslui@cse.cuhk.edu.hk (J.C.S. Lui).
}

peering link is nominally free of charge under the free peering relationship.

Under this transit service model, an ISP needs to make peering decisions (e.g. whether to peer with the other ISP or not and with what kind of peering relationship), provisioning decisions (e.g. how much capacity shall this ISP provision), and pricing decisions (e.g. how much shall this ISP charge its own subscribers) to cope with inter-ISP traffic and optimize its business.

Inter-ISP traffic has important impact on ISPs' business for at least two reasons. First, the monetary settlements between ISPs are directly based on inter-ISP traffic flows. Secondly, inter-ISP links are likely to be bottlenecks that affect the performance perceived by potential subscribers, hence indirectly affect the ISPs' market share distribution. 
Inter-ISP traffic exchanges are generated by various applications. In the current Internet, web traffic and overlay traffic are the two dominant types of traffic. BGP, the current Inter-ISP routing protocol, can help ISPs control the routing of web traffic. BGP ensures that the actual web information flows (traffic) are confined to be along the routes that are advertised between the peering ISPs according to ISPs' business considerations. So ISPs' settlement is what they expect from their negotiated peering agreements.

Such policy-based routing, however, focuses on business considerations and does not imply the best possible service for individual subscribers and the applications they are running. As a consequence, today's Internet is full of overlay applications, which change traffic routing in the application layer to better satisfy and optimize for applications' (or subscribers') needs.

Take P2P overlay networks as an example. To provide efficient and speedy distribution of content to many receivers, peers play the role of information receiver as well as server at the same time. This causes information to be routed according to the distribution of the overlay nodes (peers), and to some extent the performance of paths between the peers. The resultant patterns of information flow may be quite different from that intended by the ISPs' transit agreements. For example, let us assume that two ISPs, ISP $A$ and ISP $B$, peer with each other through a free peering link. This means neither ISP $A$ nor ISP $B$ would like to provide transit service to the Internet for each other. Suppose peers of a P2P (overlay) network populate both these two ISP networks, but ISP A's network has a large peer population who download a large number of shared objects from the Internet. Due to the nature of the P2P application (e.g. file sharing), peers in ISP $B$ can easily find shared objects from (peers in) ISP $A$ rather than from the Internet, hence fetch them from ISP A's network rather than from the Internet. As a result, ISP A's network is effectively providing transit service for ISP $B$ without compensation, which is inconsistent with the peering agreements between the two ISPs.

What are the implications of such overlay network traffic on ISPs peering? Can the ISPs still rely on Internet's policy-based routing protocol (BGP) to manage their traffic, or will they need new strategies or new Internet business model in view of the overlay traffic?

The contribution of this work is to present an inter-ISP traffic model together with game-theoretic analysis to help understand the ISP peering and provisioning issues under application layer traffic routing. The game-theoretic model is an abstract model designed to capture the decision of the major players of the Internet marketplace - the subscribers (users), and the ISPs playing different roles in providing transit service. In particular, we focus on the situation in a local market where there are two ISPs competing for subscribers and they are connected to the Internet via upstream transit ISPs. In such a model, the ISPs are modelled as leaders who optimize their profits by setting proper prices, and subscribers are modeled as followers who react to the prices set by the local ISPs. The operation of these local ISPs can be expected to reach some market equilibrium, when each ISP attaining a certain market share of the subscribers and making a certain amount of profit.
We adopt a gravitational traffic model as a simple and intuitive way to model the inter-ISP traffic patterns generated by different applications. In this model, traffic flows according to the number of subscribers in each ISP engaged in the same application at the same time, modulated by performance influence which leads to specific route selection by overlay networks. In other words, an ISP with more subscribers tends to attract (or contribute) more traffic, with the throttling of the performance of links connecting this ISP to its neighbors. By adjusting its parameters, such a gravitational model provides a rational approximation of different inter-ISP traffic patterns. Our inter-ISP traffic model pinpoints the important factors that affect interISP traffic intensities, i.e. population distribution, routing sensitivity to network performance, and routing policies which are related to ISPs' business relationship.

Our analysis brings out several interesting observations.

(i) As the traffic model shifts from traditional web traffic to more overlay traffic, the benefit of the free peering may become unfairly distributed. The smaller ISP can enjoy a certain degree of free-riding. This may trigger some actions by the bigger ISP, though the possible actions may be counter-productive (as explained below).

(ii) If the larger ISP tries to cut back the bandwidth provisioning of its private $\operatorname{link}(\mathrm{s})$ to the Internet, it will help restore the fairness of peering benefits; but this is detrimental to business expansion and is not healthy for the growth of the Internet.

(iii) If the larger ISP tries to reduce the peering bandwidth, it will also help restore the fairness of peering benefits. However, this also may hurt the ISP business since it reduces the collective bargaining power of the ISPs in setting prices for subscribers.

The insights from this work is useful for further study of ISP peering practices, and considerations for different inter-domain routing mechanisms and other tools to support these new peering practices. From a single ISP's perspective, our methodology can also be used to build a decision support system to evaluate various peering and provisioning strategies.

The rest of the paper is organized as follows. Related work is given in Section 2. In Section 3, we introduce our business model and formulate the local access market as a multi-leader-follower game. We propose our general inter-ISP traffic model based on caching function and performance sensitivity function in Section 4. Based on the game-theoretic framework and the inter-ISP traffic model, Section 5 presents our case studies for Web traffic and overlay traffic. Section 6 concludes our discussions.

\section{Related work}

Due to the inter-dependency of the economic principles and the technical architecture of the Internet operations, there has been increasing interest in applying economic and game-theoretic analysis to study Internet protocol and operational issues. For example, [20] presents a simple 
traffic model between local ISPs and uses a simple economic model to predict when these ISP would use no-payment peering rather than customer-provider peering for their local traffic. In [17], the authors give an interesting analysis of how transit and customer prices are set in a network consisting of multiple ISPs. They also examine the existence of equilibrium price strategies and show how positive profit can be achieved using threat strategies. In [8], the problem of where a pair of ISPs will make their peering connections so as to minimize their own transit traffic is formulated and studied by a game-theoretic model. Chun et al. [4] introduces a novel non-cooperative game model to characterize the caching problem among selfish servers without any central coordination and analyze replication of resources by these server nodes.

The rise of P2P overlay traffic in broadband networks has been reported and discussed academically as well as in popular press $[6,18]$. The emergence of various overlay networks raises many technical challenges and business issues for ISPs. Researchers are making efforts to understand these new traffic patterns and improve their performance $[1,2]$, and the problematic interactions between IP networks and overlay networks are examined in $[9,16]$.

In [19], we point out that the application layer routing of overlay networks upsets ISPs' traditional business model. However, that economic analysis does not take the response of subscribers into consideration, and we use break-even prices to evaluate one ISP's business, which cannot capture the stable state of one market. We also assume an ideal situation where each resource is downloaded only once by one ISP and then can be shared by all subscribers in the ISP. It is likely to be the direction of future overlay networks due to the tussle between ISPs and overlay networks (which is illustrated by those approaches mentioned in $[1,2]$ ), but it is far away from the reality of current P2P overlay traffic pattern.

\section{Business model and game theoretic framework}

We focus on the local market with $n$ homogeneous subscribers as shown in Fig. 1. The subscribers can access the Internet via two ISPs, say ISP 1 and $\mathrm{ISP}_{2}$. Each local ISP is in the business of providing access service, and competes with the other ISP for subscriber share. We denote ISP's market share as $\alpha_{i}(i=1,2)$, and $\alpha_{1}+\alpha_{2}=1$.

Both ISPs need to set up connections with their respective providers (which could be the same) for access to the rest of the Internet. In practice, many ISPs are multihomed, which means they have multiple provider links to reach the Internet. In our analysis, we use one virtual link between ISP $_{i}$ and the Internet to denote these multiple physical links and this virtual link is referred to as ISP $_{i}$ 's private link. These two local ISPs may also set up a free peering link between them to reduce traffic on their respective private links. The traffic exchange on peering links is free of charge. In addition, we ignore the peering cost such as maintenance cost in this analysis.

In the following discussion, the incoming capacity of $\mathrm{ISP}_{i}$ 's private link (from the remote Internet to $\mathrm{ISP}_{i}$ ) is

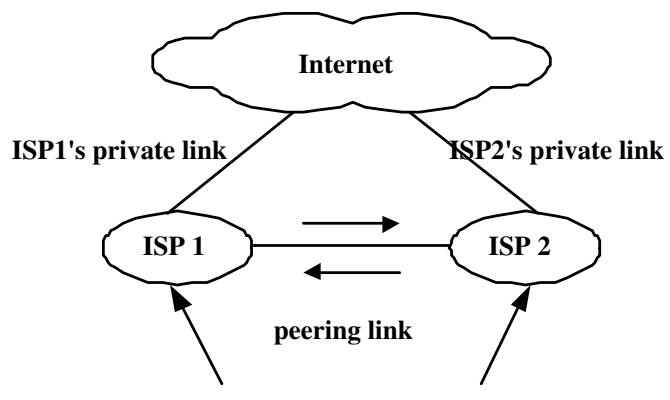

n subscribers in the local market

Fig. 1. The local market under study.

denoted by $c_{i}^{\mathrm{r}}$, and the traffic intensity on $\mathrm{ISP}_{i}$ 's private links (from the remote Internet to $\mathrm{ISP}_{i}$ ) is denoted by $t_{i}^{\mathrm{r}}$. Similarly, the incoming capacity of ISP $_{i}$ 's peering link (from the peering ISP to $\operatorname{ISP}_{i}$ ) is denoted by $c_{i}^{\mathrm{p}}$, and the traffic intensity on that link is denoted by $t_{i}^{\mathrm{p}}$.

Let $D_{i}$ denote the total amount of ISP,'s subscribers's demand. Obviously we have $t_{i}^{\mathrm{r}}+t_{i}^{\mathrm{p}}<D_{i}$, which means some of the demand is satisfied by peers or servers in ISP' ${ }_{i}$ 's local network. Let $t_{i}^{1}$ denote the demand intensity that is satisfied locally, and then we have $t_{i}^{\mathrm{l}}=D_{i}-t_{i}^{\mathrm{r}}-t_{i}^{\mathrm{p}}$.

\subsection{Business model}

Obviously, each subscriber needs to pay its provider for the access service. As it is quite common with current ISP pricing, we assume that each ISP uses flat rate charging and charges a fixed price $p_{i}$, and it is possible that $p_{1} \neq p_{2}$.

Local ISPs pay their providers for using their transit service. We assume that the transit providers charge local ISPs in the same local market, (i.e. ISP $_{1}$ and $\mathrm{ISP}_{2}$ ), in the same way. The cost incurred is computed based on the amount of traffic a customer ISP generates and the performance of the link between two ISPs, i.e. cost $=f(t, c)$, where $t$ is a variable determined by the customer ISP's traffic volume, $c$ is related to the capacity of the inter-ISP links and $f(\cdot)$ is a non-decreasing function that maps $t$ and $c$ to cost.

Various charging models differ from one another in their choices of $c, t$ and the cost function $f(\cdot)$. For example, $t$ can be the total-volume of traffic one customer ISP generates during the entire charging period, which is referred to as "total-volume based charging"; or $t$ can be the 95th percentile of all 5-minute traffic volumes during the charging period, which is called "percentile-based charging". In this paper, we are not going to study the traffic dynamics on a short time scale, which implies that $t$ can be the average traffic intensity on the inter-ISP links for our purpose.

Although $c$ does not usually appear directly in the cost function, it in fact affects the cost which can be seen from the comparison that a traffic intensity of $40 \mathrm{Mbps}$ on a OC3 link must cost more than a traffic intensity of $40 \mathrm{Mbps}$ on a DS3 link.

The cost function $f(\cdot)$ can be quite complicated in the real world, for example, a piece-wise linear (nondecreasing) function. 


\subsection{Game formulation}

In this subsection, we will explain our game formulation for the network in Fig. 1. In our game formulation, local ISPs have determined the capacity of the links in the network, i.e. $c_{i}^{\mathrm{r}}$ and $c_{i}^{\mathrm{p}}$ are fixed. The game is to model the interaction of two ISPs and subscribers in this market, and find out the market share $\alpha_{i}$ and the optimum price $p_{i}$ when the game for this market reaches Nash Equilibrium. Later we will analyze Nash Equilibria of games with different $c_{i}^{\mathrm{r}}$ and $c_{i}^{\mathrm{p}}$ to study ISPs' peering strategies and provisioning strategies.

In the local market, ISPs set the prices for their own subscribers independently, and each subscriber responds to the prices by choosing one ISP as its provider to optimize its own utility. It is naturally a two-stage multi-leader-follower game [15], in which ISPs act as leaders, with $n$ subscribers acting as followers that play a game with the prices $p_{i}(i=1,2)$ as ISP strategies.

Similar to the analysis of a Stackelberg game, we first analyze the second stage of this game, assuming that ISP $_{i}(i=1,2)$ has set the price $p_{i}$ at the first stage of the game. All subscribers in this game share the same utility function, which means they choose their individual providers according to the same rules.

The most important concerns of one subscriber are access cost $\left(p_{i}\right.$ if choose $\operatorname{ISP}_{i}$ and service performance. Since one ISP is with fixed inter-ISP link capacity, its service performance will become worse as it provides access service to more subscribers. Let $P_{i}\left(\alpha_{i}\right)$ denote the service performance of ISP $P_{i}$ when it provides access service to $n \alpha_{i}$ subscribers. Obviously $P_{i}(\cdot)$ is a decreasing function and $P_{1}(\cdot)$ may not be the same as $P_{2}(\cdot)$ because two ISPs may have different inter-ISP link capacity.

Let $\mathscr{U}_{i}\left(p_{i}, \alpha_{i}\right)$ denote the utility value of subscribers in ISP $_{i}$ when ISP $P_{i}$ provides service for $n \alpha_{i}$ subscribers at a price of $p_{i}$. We define

$\mathscr{U}_{i}\left(p_{i}, \alpha_{i}\right)=P_{i}\left(\alpha_{i}\right)-p_{i}$,

which shows that subscribers always prefer lower access cost and better service performance. Since all subscribers of one ISP are with the same utility value, we also refer to $\mathscr{U}_{i}$ as $\mathrm{ISP}_{i}$ 's service utility.

Subscribers are not required to make their decisions simultaneously, which implies that we consider the Nash Equilibrium of the second stage game as a stable state where all subscribers settle down and will not change their decisions. Let $\alpha_{1}^{*}$ be ISP ${ }_{1}$ 's market share at the Nash Equilibrium point. After all subscribers in the local market finish selecting their ISPs, we have

$\mathscr{U}_{1}\left(p_{1}, \alpha_{1}^{*}\right)=\mathscr{U}_{2}\left(p_{2}, 1-\alpha_{1}^{*}\right)$,

which means the utility of all subscribers in both ISPs evaluate to the same value, hence none of them has a reason to change his decision and thus the market becomes stable.

Note that Eq. (2) may not hold for some extreme cases with $\alpha_{1}^{*}=0$ or $\alpha_{1}^{*}=1$. For example, $\mathscr{U}_{1}\left(p_{1}, 1\right)>\mathscr{U}_{2}\left(p_{2}, 0\right)$ implies ISP $_{1}$ grabs all subscribers and it still can provide a better utility for new subscribers. This is a degenerate case where one ISP dies. In the following analysis, we ignore such degenerate cases, and assume Eq. (2) always holds for Nash Equilibrium of the game.

Let us define $\varphi(\alpha)=P_{1}(\alpha)-P_{2}(1-\alpha)$. From Eqs. (1) and (2), we have

$p_{1}-p_{2}=P_{1}\left(\alpha_{1}^{*}\right)-P_{2}\left(1-\alpha_{1}^{*}\right)=\varphi\left(\alpha_{1}^{*}\right)$

We assume that the two local ISPs together have the full control of the market by setting the prices, which is equivalent to assuming that $\varphi(\cdot)$ is an invertible function. Then we can solve the second stage of our game and the Nash Equilibrium is

$\alpha_{1}^{*}\left(p_{1}, p_{2}\right)=\varphi^{-1}\left(p_{1}-p_{2}\right)$.

In summary, the second stage game takes prices set by ISPs (i.e. $p_{1}$ and $\left.p_{2}\right)$ as input and gives $\alpha_{1}^{*}\left(p_{1}, p_{2}\right)$ as outcome. $\alpha_{i}^{*}\left(p_{1}, p_{2}\right)$ reflects the rational response of subscribers to the prices $p_{1}$ and $p_{2}$.

At the first stage, the ISPs do not cooperate among themselves because they are competing with each other for market share. They need to set their prices (in a one-shot game) simultaneously and independently, which implies the first stage game is a standard Nash game. The action set of $\mathrm{ISP}_{i}$ in this non-cooperate game is $p_{i} \geqslant 0$. We define a utility function $\mathscr{R}_{i}$, where $\mathscr{R}_{i}\left(p_{1}, p_{2}\right)$ is the preference of $\operatorname{ISP}_{i}$ to an action profile $\left(p_{1}, p_{2}\right) . \mathscr{R}_{i}\left(p_{1}^{\prime}, p_{2}^{\prime}\right)>$ $\mathscr{R}_{i}\left(p_{1}^{\prime \prime}, p_{2}^{\prime \prime}\right)$ means ISP $i$ prefers the profile $\left(p_{1}^{\prime}, p_{2}^{\prime}\right)$ to $\left(p_{1}^{\prime \prime}, p_{2}^{\prime \prime}\right)$.

We assume that one ISP simply uses its profit (revenue minus cost) to evaluate a profile. ISPs, acting as leaders of the multi-leader-follower game, are aware of the function $\alpha_{i}^{*}\left(p_{1}, p_{2}\right)$, the rational reaction of subscribers to prices. Therefore the utility function is defined as follows:

$\mathscr{R}_{i}\left(p_{1}, p_{2}\right)=p_{i} n \alpha_{i}^{*}\left(p_{1}, p_{2}\right)-f\left(t_{i}^{\mathrm{r}}\left(\alpha_{i}^{*}\left(p_{1}, p_{2}\right)\right), c_{i}^{\mathrm{r}}\right)$.

Here, $t_{i}^{\mathrm{r}}$, the traffic intensity on $\mathrm{ISP}_{i}$ 's private link, is certainly related to ISP $_{i}$ 's subscriber share, so we write it in the form of $t_{i}^{\mathrm{r}}\left(\alpha_{i}^{*}\left(p_{1}, p_{2}\right)\right)$.

From Eq. (3), we can see that the market share distribution would not change if two ISPs agree to increase their prices at the same time, and then both ISPs can increase their revenues. However, two ISPs are not cooperating with each other, and both of them have incentives to lower their individual price to attract subscribers. In the numerical studies, we will show that peering helps two ISPs cooperate with each other to some extent and it results in higher prices.

As a summary, the multi-leader-follower game played by two ISPs and subscribers gives rise to the following optimization problem:

$$
\begin{array}{ll}
\max _{p_{i} \geqslant 0} & \mathscr{R}_{i}\left(\widehat{p}_{i}\right), \quad i=1,2 \\
\text { subject to } & \alpha_{1}^{*}\left(p_{1}, p_{2}\right)=\varphi^{-1}\left(p_{1}-p_{2}\right), \\
\text { where } \widehat{p}_{1}=\left(p_{1}, p_{2}^{*}\right) \text { and } \widehat{p}_{2}=\left(p_{1}^{*}, p_{2}\right) .
\end{array}
$$

We give an overview of the game theoretic framework to study this market in Fig. 2 . For any given $p_{1}$ and $p_{2}$, subscribers play a game as $n$ followers and yield the value of $\alpha_{i}$ as an outcome, which is reflected in the constraint in Eq. (5). This is the second stage of the whole game, expressed as the bigger ellipse in the figure.

$\mathrm{ISP}_{1}$ and $\mathrm{ISP}_{2}$, acting as the leaders of this two-stage multi-leader-follower game, can predict the response $\alpha_{i}$ 


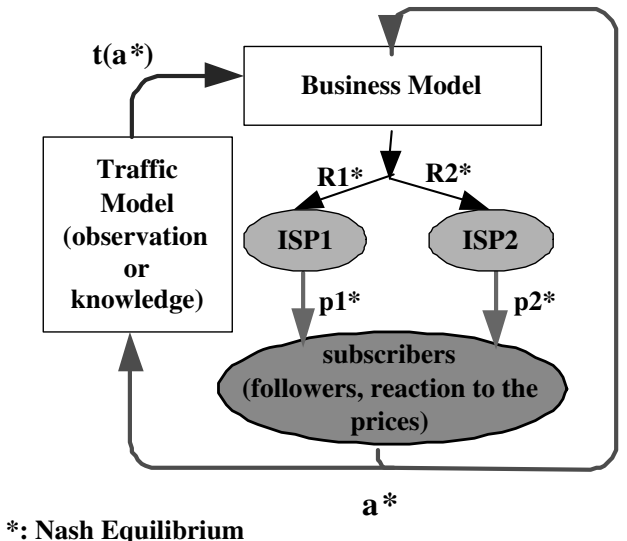

Fig. 2. Overview of the game theoretic framework.

under different profiles $\left(p_{1}, p_{2}\right)$, i.e. they know the function $\alpha_{1}^{*}\left(p_{1}, p_{2}\right)$.

ISPs' knowledge of the relation between traffic intensities on different inter-ISP links and the market share distribution is expressed as the traffic model. This model accepts the market share distribution $\alpha_{i}^{*}$ as an input (the most leftbottom arrow), and outputs traffic intensities on different inter-ISP links, i.e. $t\left(\alpha_{i}^{*}\right)$ (the most left-top arrow). The traffic model will be further discussed in Section 4.

ISPs' knowledge of the dependence of their profits on the market share distribution and traffic intensity distribution is expressed as the business model. This model accepts the market share distribution (related to ISPs' revenues) and the traffic intensity distribution (related to ISPs' costs) as inputs (the two top arrows), and gives ISPs' profits as its output. The business model is discussed in Subsection 3.1 and then mathematically expressed as Eq. (4).

As a result of ISPs' knowledge of subscribers' response, traffic model and business model, ISPs can eventually predict their own profits ( $\mathscr{R}_{1}$ and $\left.\mathscr{R}_{2}\right)$ under different profiles $\left(p_{1}, p_{2}\right)$. Therefore, two ISPs that are playing a non-cooperate game are able to maximize their own profits by making optimum decisions on their own prices, which is reflected in the objective function in Eq. (5). This is the first stage of the whole game and the interaction of two ISPs gives $\left(p_{1}^{*}, p_{2}^{*}\right)$ as the Nash Equilibrium which represents the steady state of the market.

\subsection{Game solution}

As an extension of the Nash game which models the situation where each player takes no leadership position over its rivals, the multi-leader-follower game arises to analyze the situation where one or more players have more power than other players and thus become leader(s) in the game. The simplest of multi-leader-follower game is the Stackelberg game in which there is only one leader and multiple followers. A mathematical model for the Stackelberg game is the MPEC (mathematical programs with equilibrium constraints). The computation of global solutions to MPECs remains elusive, if not impossible, regardless of the progress in research on the MPEC $[5,14]$.
A multi-leader-follower game with multiple leaders is more complicated than a Stackelberg game. The existence of Nash Equilibrium for a multi-leader-follower game is in jeopardy. Even in the favorable case where such an equilibrium exists, its complete characterization remains a daunting, if not impossible, task. Any rigorous attempt to compute a Nash Equilibrium (if it exists) for a multi-leader-follower game is presently out of the reach of existing methods, due to the high level of complexity and technical difficulties $[10,15]$.

Fortunately, the game in this paper takes specific utility functions $\mathscr{U}_{i}$ and $\mathscr{R}_{i}$, so that we are able to do a further study on its Nash Equilibrium. First, we rewrite Eq. (5) as follows:

$$
\begin{cases}p_{1}^{*}=\operatorname{argmax}_{p_{1}} & p_{1} n \alpha_{1}\left(p_{1}, p_{2}^{*}\right)-f\left(t_{1}^{\mathrm{r}}\left(\alpha_{1}\left(p_{1}, p_{2}^{*}\right)\right), c_{1}^{\mathrm{r}}\right), \\ p_{2}^{*}=\operatorname{argmax}_{p_{2}} & p_{2} n \alpha_{2}\left(p_{1}^{*}, p_{2}\right)-f\left(t_{2}^{\mathrm{r}}\left(\alpha_{2}\left(p_{1}^{*}, p_{2}\right)\right), c_{2}^{\mathrm{r}}\right), \\ \text { subject to } & p_{1}-p_{2}=\varphi\left(\alpha_{1}\right) .\end{cases}
$$

We have the following theorem on the necessary condition for a Nash Equilibrium of the game defined in the above equation:

Theorem 1. Define

$$
\begin{aligned}
& \Phi_{1}\left(\alpha_{1}\right)=\varphi\left(\alpha_{1}\right)+\alpha_{1} \frac{\partial \varphi}{\partial \alpha_{1}}-\frac{1}{n} \frac{\partial f\left(c_{1}\right)}{\partial t_{1}^{\mathrm{r}}} \frac{\partial t_{1}^{\mathrm{r}}}{\partial \alpha_{1}}, \\
& \Phi_{2}\left(\alpha_{1}\right)=\varphi\left(\alpha_{1}\right)+\left(\alpha_{1}-1\right) \frac{\partial \varphi}{\partial \alpha_{1}}-\frac{1}{n} \frac{\partial f\left(c_{2}\right)}{\partial t_{2}^{\mathrm{r}}} \frac{\partial t_{2}^{\mathrm{r}}}{\partial \alpha_{1}} .
\end{aligned}
$$

For $\alpha_{1}^{*}$ to be a Nash Equilibrium of the multi-leader-follower game defined in Eq. (6), it must satisfy the following two conditions:

$$
\begin{array}{ll}
\text { (i). } & \Phi_{1}\left(\alpha_{1}^{*}\right)+\Phi_{2}\left(\alpha_{1}^{*}\right)-\varphi\left(\alpha_{1}^{*}\right)=0, \\
\text { (ii). } & \left.\frac{\partial \Phi_{1}}{\partial \alpha_{1}}\right|_{\alpha_{1}^{*}}<0,\left.\quad \frac{\partial \Phi_{2}}{\partial \alpha_{1}}\right|_{\alpha_{1}^{*}}<0 .
\end{array}
$$

Proof 1. Assume $\alpha_{1}^{*}$ is a Nash Equilibrium that is yielded from $\left(p_{1}^{*}, p_{2}^{*}\right)$. Obviously we have $p_{1}^{*}-p_{2}^{*}=\varphi\left(\alpha_{1}^{*}\right)$.

From the definition of Nash Equilibrium, we know that $p_{1}^{*}$ should be the best response to $p_{2}^{*}$, and vice versa. Since we do not consider the cases where the maximum profit happens at the boundary, we must have

$$
\begin{gathered}
\left.\frac{\partial \mathscr{R}_{1}\left(p_{1}, p_{2}^{*}\right)}{\partial p_{1}}\right|_{p_{1}^{*}}=0,\left.\quad \frac{\partial R_{1}^{2}\left(p_{1}, p_{2}^{*}\right)}{\partial p_{1}^{2}}\right|_{p_{1}^{*}}<0, \\
\left.\frac{\partial \mathscr{R}_{2}\left(p_{1}^{*}, p_{2}\right)}{\partial p_{2}}\right|_{p_{2}^{*}}=0,\left.\quad \frac{\partial R_{2}^{2}\left(p_{1}^{*}, p_{2}\right)}{\partial p_{2}^{2}}\right|_{p_{2}^{*}}<0 .
\end{gathered}
$$

Moreover, because $\alpha_{1}=\varphi^{-1}\left(p_{1}, p_{2}\right)$ exists, we have

$$
\begin{aligned}
& \left.\frac{\partial \mathscr{R}_{1}\left(\alpha_{1}, p_{2}^{*}\right)}{\partial \alpha_{1}}\right|_{\alpha_{1}^{*}}=\left.\left.\frac{\partial \mathscr{R}_{1}\left(p_{1}, p_{2}^{*}\right)}{\partial p_{1}}\right|_{p_{1}^{*}} \cdot \frac{\partial p_{1}}{\partial \alpha_{1}}\right|_{\alpha_{1}^{*}}, \\
& \left.\frac{\partial p_{1}}{\partial \alpha_{1}}\right|_{\alpha_{1}^{*}}=\left.\frac{\partial \varphi\left(\alpha_{1}\right)}{\partial \alpha_{1}}\right|_{\alpha_{1}^{*}} \neq 0 .
\end{aligned}
$$

We can apply this property on all conditions in Eq. (8) and rewrite them as follows: 
$\left.\frac{\partial \mathscr{R}_{1}\left(\alpha_{1}, p_{2}^{*}\right)}{\partial \alpha_{1}}\right|_{\alpha_{1}^{*}}=0,\left.\quad \frac{\partial \mathscr{R}_{2}\left(\alpha_{1}, p_{1}^{*}\right)}{\partial \alpha_{1}}\right|_{\alpha_{1}^{*}}=0$,
$\left.\frac{\partial \mathscr{R}_{1}^{2}\left(\alpha_{1}, p_{2}^{*}\right)}{\partial \alpha_{1}^{2}}\right|_{\alpha_{1}^{*}}<0,\left.\quad \frac{\partial \mathscr{R}_{2}^{2}\left(\alpha_{1}, p_{1}^{*}\right)}{\partial \alpha_{1}^{2}}\right|_{\alpha_{1}^{*}}<0$.

From Eqs. (9), (4) and $p_{1}^{*}-p_{2}^{*}=\varphi\left(\alpha_{1}^{*}\right)$, we derive that

$$
\begin{gathered}
p_{2}^{*}+\varphi\left(\alpha_{1}^{*}\right)+\left.\alpha_{1}^{*} \frac{\partial \varphi}{\partial \alpha_{1}}\right|_{\alpha_{1}^{*}}-\left.\frac{1}{n} \frac{\partial f\left(c_{1}^{\mathrm{r}}\right)}{\partial t_{1}^{\mathrm{r}}} \frac{\partial t_{1}^{\mathrm{r}}}{\partial \alpha_{1}}\right|_{\alpha_{1}^{*}}=0, \\
-p_{2}^{*}+\left.\left(\alpha_{1}^{*}-1\right) \frac{\partial \varphi}{\partial \alpha_{1}}\right|_{\alpha_{1}^{*}}-\left.\frac{1}{n} \frac{\partial f\left(c_{2}^{\mathrm{r}}\right)}{\partial t_{2}^{\mathrm{r}}} \frac{\partial t_{2}^{\mathrm{r}}}{\partial \alpha_{1}}\right|_{\alpha_{1}^{*}}=0 .
\end{gathered}
$$

It can be further simplified based on Eq. (7) as follows:

$$
\begin{aligned}
& p_{2}^{*}+\Phi_{1}\left(\alpha_{1}^{*}\right)=0, \\
& -\varphi\left(\alpha_{1}^{*}\right)-p_{2}^{*}+\Phi_{2}\left(\alpha_{1}^{*}\right)=0 .
\end{aligned}
$$

$\alpha_{1}^{*}$ must satisfy the following condition in order to be the market share of a Nash Equilibrium:

$\Phi_{1}\left(\alpha_{1}^{*}\right)+\Phi_{2}\left(\alpha_{1}^{*}\right)-\varphi\left(\alpha_{1}^{*}\right)=0$.

Eq. (10) can be simplified as

$\left.\frac{\partial \Phi_{1}}{\partial \alpha_{1}}\right|_{\alpha_{1}^{*}}<0,\left.\quad \frac{\partial \Phi_{2}}{\partial \alpha_{1}}\right|_{\alpha_{1}^{*}}<0$.

This theorem gives us a way to compute the Nash Equilibrium of the game which represents the steady state of the local market. If the traffic function $t_{i}^{\mathrm{r}}\left(\alpha_{i}\right)$ takes a simple form, we can derive the Nash Equilibrium in closed-form directly from this theorem. The $\alpha_{1}^{*}$ can be solved using Eq. (12), $p_{1}^{*}$ and $p_{2}^{*}$ can be derived from $p_{2}^{*}=-\Phi_{1}\left(\alpha_{1}^{*}\right)$ and $p_{1}^{*}=\Phi_{2}\left(\alpha_{1}^{*}\right)$. And then we can further study various issues in this local market based on this closed-form game solution. Unfortunately, as we will discuss in later sections, the traffic function in real world is likely to be very complicated, which makes closed-form game solution impossible. In this paper, we will study the local market numerically using Matlab to avoid solving the fix-point problem in the traffic model, but the Matlab code is still based on this theorem.

Eq. (12) shows that the traffic pattern $t_{i}^{\mathrm{r}}\left(\alpha_{i}\right)$ affects the Nash Equilibrium. In the next section, we will present our traffic model as a simple and intuitive way to represent inter-ISP traffic patterns. Then we will show how our framework can help ISPs make peering and provisioning decisions in the context of different traffic patterns.

\section{Inter-ISP traffic model}

The inter-ISP traffic is a significant factor that determines the outcome of the ISP peering game, for at least two reasons. First, the monetary flows among ISPs are directly based on inter-ISP traffic flows. Secondly, inter-ISP links are likely to be bottlenecks that affect the performance perceived by potential subscribers, hence indirectly affect the ISPs' market share distribution. Therefore $t_{i}^{\mathrm{r}}$ appears in both subscribers' utility function ' $\mathscr{U}_{i}$ and ISPs' utility function $\mathscr{R}_{i}$. On the other hand, it is very difficult to characterize real world traffic patterns using simple traffic models. This is usually the most contentious part of modeling efforts.

In this paper, we describe a reasonably simple yet general traffic model $t(\alpha)$, focusing on our problem at hand, namely describing the internal and external traffic of a set of local ISPs based on their market share distribution $\alpha$ and their network provisioning.

Fig. 3 is an illustration of all factors that affect the intensity of inter-ISP traffic. In a network, both ISPs' behavior and subscribers' behavior have influence on traffic intensities on different links. ISPs decide the provisioning of their links $\left(c_{i}^{\mathrm{r}}\right.$ and $\left.c_{i}^{\mathrm{p}}\right)$, and they also implement their routing rules using BGP. The market share distribution $(\alpha)$ is determined by subscribers' choice of their own providers, and subscribers may also affect the routing of the traffic using application layer routing. The traffic model takes all these factors as input and predicts traffic intensities on interISP links as output. The traffic model shows the reaction of the network to the behavior of the ISPs and subscribers.

\subsection{Gravity modeling framework}

We assume that subscribers in this market are homogeneous, and each subscriber generates traffic demand with an intensity of $\rho$. Therefore the total demand of ISP,'s subscribers is $D_{i}=n \rho \alpha_{i}$. So we have

$t_{i}^{\mathrm{r}}+t_{i}^{\mathrm{p}}+t_{i}^{\mathrm{l}}=n \rho \alpha_{i}, \quad i=1,2$.

The task of our traffic model can be seen as to assign the total demand to three different categories - local intraISP traffic $\left(t_{i}^{1}\right)$, peering traffic $\left(t_{i}^{\mathrm{p}}\right)$ and private traffic $\left(t_{i}^{\mathrm{r}}\right)$. We solve this problem using gravity model as a reference.

Gravity models are commonly used by social scientists to model the movement of people, goods or information between geographic areas. Generally, those models contain some elements of mass and distance, which lends them to the metaphor of physical gravity as described in Newton's law of gravity. For example, in gravity models for cities, the relative strength of the interaction between two cities might be modeled as directly proportional to the product of the populations and inversely proportional to the distance between the two cities. Recently, researchers start to use gravity model to solve some networking issues such as the estimation of inter-ISP demand matrix [3] and intra-ISP demand matrix [21].

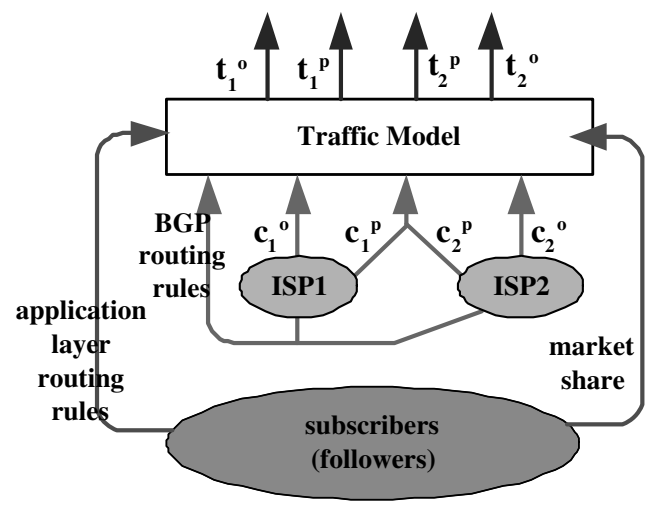

Fig. 3. Overview of the traffic model. 
A general formulation of a gravity model is given by the following equation:

$t_{i}^{j} \propto \frac{S_{j} \times D_{i}}{F_{i}^{j}}$,

wherein

(i) $t_{i}^{j}$ is a variable under study that is related to the flow from $j$ to $i$.

(ii) $S_{j}$ represents the repulsive factor that is associated with "leaving" from $j$.

(iii) $D_{i}$ represents the attractive factor that is associated with "going" to $i$.

(iv) $F_{i}^{j}$ is a distance factor that is related to the path from $j$ to $i$.

$S_{j}, D_{i}$ and $F_{i}^{j}$ should be interpreted appropriately when the gravity model is applied in different contexts under study. In [21], $t_{i}^{j}$ is the traffic volume that enters the network at location $j$ and exits at location $i$. $S_{j}$ is interpreted as the traffic volume entering the network at location $j$, and $D_{i}$ is interpreted as the traffic volume exiting at location $i . F_{i}^{j}$ is simply assumed to be a common constant that does not depend on $i$ and $j$. In [3], $t_{i}^{j}$ is the inter-ISP traffic volume from ISP ${ }_{j}$ to $\operatorname{ISP}_{i}$. The attractive factor and repulsive factor are interpreted as the population size of one ISP's subscribers $\left(P_{\mathrm{RA}}(\cdot)\right)$ or the size of one ISP's web contents $\left(P_{\text {web }}(\cdot)\right)$. And $F_{i}^{j}$ is the transit quality of the bottleneck ISP in the given path between $\operatorname{ISP}_{i}$ and $\operatorname{ISP}_{j}$.

The model in [3] is to estimate the intensity of the demand between any pair of ISPs, while the routing of this demand is not considered. We agree that population size and path performance are the most important factors that affect inter-ISP traffic intensity. However, that model cannot serve our purpose to predict the inter-ISP traffic intensity for two reasons. First, ISPs may deploy various approaches to improve the locality of applications and reduce inter-ISP traffic, so subscribers are more likely to connect with other subscribers (or servers) in the same ISP network to retrieve information. Therefore, the attractive factor and the repulsive factor may not be linearly dependent on the population size of one ISP. Second, we need to predict traffic intensity on the inter-ISP links between neighboring ISPs instead of demand intensity between any pair of ISPs, thus routing must be included. Therefore we interpret the gravity model in a slightly different way.

To make it easy to read in our context, we rewrite Eq. (14) as follows:

$t_{i}^{\#} \propto S_{i}^{\#} \times D_{i} \times G_{i}^{\#}, \quad \#=l, r, p$.

Note it is not a rigorous gravity model since $S_{i}^{\#}$ depends on the source ISP as well as the destination ISP in order to reflect the locality feature of P2P applications. From Eqs. (13) and (15), we have

$t_{i}^{\#}=\frac{S_{i}^{\#} \times G_{i}^{\#}}{S_{i}^{\mathrm{r}} G_{i}^{\mathrm{r}}+S_{i}^{\mathrm{p}} G_{i}^{\mathrm{p}}+S_{i}^{\mathrm{l}} G_{i}^{\mathrm{l}}} \cdot n \rho \alpha_{i}$,

where $G_{i}^{\#}$ is referred to as performance factor (inverse of distance factor). $S_{i}^{\#}$ and $G_{i}^{\#}$ will be interpreted in the following subsections.

\subsection{Interpretation of performance factor}

The performance factor $G_{i}^{\#}$ is used to model the effect of application layer routing which is sensitive to link performance.

As we know, the routing of web traffic is controlled by ISPs using BGP. The traffic demand between $\operatorname{ISP}_{i}$ and the Internet is transited via the ISP ${ }_{i}$ 's private link, and the traffic demand between two peering ISPs is transited via the peering link. The routing of web traffic does not usually matter with the inter-ISP link performance.

However, the application layer routing of overlay networks is determined by applications and it is usually sensitive to link performance. Generally speaking, overlay networks attempt to provide "enhanced" services to applications by routing their traffic according to some performance constraints. A degradation in the performance of one path will trigger overlay networks to find an alternate path that satisfies the performance constraints and reroute the traffic accordingly. Although various applications or content distribution networks (CDNs) rely on different peer selection algorithms or service redirection mechanisms, they share the common feature that such mechanisms are more likely to redirect the access to other alternatives if the provisioning is poor across a certain ISP boundary. That is why some ISPs try to use bandwidth throttling approach to reduce the inter-ISP traffic intensity.

In our gravity traffic model, we use the performance factor $G_{i}^{\#}$ to model the sensitivity of the routing to the inter-ISP link performance. We define the performance sensitivity function $G(\cdot)$ and let

$G_{i}^{\#}=G\left(c_{i}^{\#}-t_{i}^{\#}\right) \quad \#=r, p$.

For web traffic we have $G(\cdot)=1$, which means the routing of web traffic cannot be affected by link provisioning. For overlay traffic, $G(\cdot)$ should be an increasing function, which implies application layer routing always favor the path with good performance. For ease of the presentation, we further assume that $0<G(\cdot) \leqslant 1$, and $G(\cdot)$ can be viewed as the throttling effect of the link provisioning. We also assume that $G_{i}^{l}=1$ for $i=1,2$. This implies that intra-ISP links always have better performance than inter-ISP links.

\subsection{Interpretation of repulsive factor}

Subscribers in $\mathrm{ISP}_{i}$ retrieve information from different source ISPs including their own network. Intuitively, larger ISPs are more likely to provide more information for these subscribers since larger ISPs may have more content servers (web traffic) and more peers that are interested in the same files as subscribers in ISP $_{i}$ (overlay traffic). The repulsive factor $S_{i}^{\#}$ is used to model the relationship between the amount of one source ISP's contribution and its population size.

In some works people assume that the amount of one source ISP's contribution is proportional to its population size. However, many mechanisms deployed by ISPs to improve the locality of traffic make the assumption of proportional contribution unsuitable for current Internet. For example, ISPs deploy various cache servers to make web contents close to their subscribers to reduce transmission 
cost. After P2P applications became popular, researchers also propose approaches, such as gateway peers and biased neighbor selection [2], to make more use of local network instead of going to other ISP networks. With the biased neighbor selection approach, the trackers are modified so that the neighbor list of a subscriber $A$, which is returned by these modified trackers, are more likely to include peers in $A$ 's own network, while previous trackers return neighbor list randomly without consideration of peer locality. The interISP traffic intensity can be tuned easily by setting different control parameters with the new tracker algorithm.

An extensive investigation is necessary to understand how these mechanisms quantitatively affect inter-ISP traffic intensities. In this paper, we only provide a framework to model the influence of population size based on a general concept of caching function.

We define caching function $r(\alpha)$ as follows. For web traffic, $r(\alpha)$ is the probability that a subscriber's request can be satisfied by its ISP's local network when the subscriber's ISP is with a market share of $\alpha$. For P2P traffic, $r(\alpha)$ is related to the neighbor selection algorithm of the P2P application, and it can be seen as the average ratio of local peers in the neighbor list of one peer in an ISP network with a market share of $\alpha$. In other words, $r(\alpha)$ determines the application layer topology of a P2P network, while the transmission rate on links of this network is further determined by $G_{i}^{\#}$.

$r(\alpha)$ is usually a non-decreasing function of $\alpha$, the market share (or population size) of the ISP in question, satisfying $0 \leqslant r(\alpha) \leqslant 1$. Note, the caching effect can be achieved either by some kind of proxy server (so that other subscribers downloading an object already downloaded previously can be satisfied locally) or by P2P applications with peers in the same ISP serving the role of a proxy server. When $r=0$, all subscriber requests result in inter-ISP traffic, whereas when $r=1$, all subscriber requests can be satisfied locally.

Specially, $r(1)$ is the probability that subscribers in this local market connect with servers (peers) in the local market (both ISPs) for content. Note that $r(1) \neq 1$ if there are servers (peers) in remote ISPs, which means it is always possible that local subscribers connect with remote servers or peers (in the Internet, but not in local market) for content. Also, $r(1)-r\left(\alpha_{i}\right)$ can be seen as the probability that subscribers in $\operatorname{ISP}_{i}$ connect with servers (peers) in the peering ISP for contents. So the repulsive factor $S_{i}^{\#}$ is defined as follows:

$S_{i}^{\mathrm{r}}=1-r(1)$,

$S_{i}^{\mathrm{p}}=r(1)-r\left(\alpha_{i}\right)$

$S_{i}^{\mathrm{l}}=r\left(\alpha_{i}\right)$.

If there is no peering link between two local ISPs, the repulsive factor is

$S_{i}^{\mathrm{r}}=1-r\left(\alpha_{i}\right)$,

$S_{i}^{1}=r\left(\alpha_{i}\right)$.

\subsection{Inter-ISP traffic function}

The combination of caching function $r(\cdot)$ and performance sensitivity function $G(\cdot)$ together define a traffic pattern. From Eqs. (16), (17) and (19), if there is no peering link between two ISPs, we simply have

$t_{i}^{\mathrm{r}}=\frac{\left(1-r\left(\alpha_{i}\right)\right) G\left(c_{i}^{\mathrm{r}}-t_{i}^{\mathrm{r}}\right)}{\left(1-r\left(\alpha_{i}\right)\right) G\left(c_{i}^{\mathrm{r}}-t_{i}^{\mathrm{r}}\right)+r\left(\alpha_{i}\right)} \cdot n \rho \alpha_{i}$.

For the scenario where there is a peering link between the two local ISPs, we have

$$
\begin{aligned}
t_{i}^{\mathrm{r}} & =\frac{r_{i}^{\mathrm{r}}\left(\alpha_{i}\right)}{r_{i}^{\mathrm{r}}\left(\alpha_{i}\right)+r_{i}^{\mathrm{p}}\left(\alpha_{i}\right)+r_{i}^{\mathrm{l}}\left(\alpha_{i}\right)} \cdot n \rho \alpha_{i}, \\
t_{i}^{\mathrm{p}} & =\frac{r_{i}^{\mathrm{p}}\left(\alpha_{i}\right)}{r_{i}^{\mathrm{r}}\left(\alpha_{i}\right)+r_{i}^{\mathrm{p}}\left(\alpha_{i}\right)+r_{i}^{\mathrm{l}}\left(\alpha_{i}\right)} \cdot n \rho \alpha_{i},
\end{aligned}
$$

where

$$
\begin{aligned}
r_{i}^{\mathrm{r}}\left(\alpha_{i}\right) & =(1-r(1)) \cdot G\left(c_{i}^{\mathrm{r}}-t_{i}^{\mathrm{r}}\right), \\
r_{i}^{\mathrm{p}}\left(\alpha_{i}\right) & =\left(r(1)-r\left(\alpha_{i}\right)\right) \cdot G\left(c_{i}^{\mathrm{p}}-t_{i}^{\mathrm{p}}\right), \\
r_{i}^{\mathrm{l}}\left(\alpha_{i}\right) & =r\left(\alpha_{i}\right) .
\end{aligned}
$$

Since overlay routing might be load-dependent, Eqs. (20) and (21) are fixed point problems and the closed-form solution cannot be given easily. In this work, we solve the problems numerically using Matlab.

\section{Case studies of ISPs peering game}

In Section 3 we define the ISPs peering game and formulate the game as an optimization problem in Eq. (6). Since inter-ISP traffic pattern play an essential role in the peering game, we further present our traffic model to describe different traffic patterns in Section 4. Based on the whole framework, now we can analyze the local market and study ISPs' provisioning and peering decisions in the context of different traffic patterns. In this section, we will do a few numerical case studies to gain some insights into how traffic patterns affect ISPs' business strategies.

In all of our case studies, we focus on the same market with the following information. There are $n=1000$ subscribers in the market, and each subscriber is with a demand of $\rho=0.001$. The cost function for local ISPs is

$f\left(c_{i}^{\mathrm{r}}, t_{i}^{\mathrm{r}}\right)=q_{\mathrm{c}} \times c_{i}^{\mathrm{r}}+q_{\mathrm{t}} \times t_{i}^{\mathrm{r}}$,

where $q_{\mathrm{t}}$ is a constant cost for per unit intensity of traffic and $q_{\mathrm{c}}$ is a constant cost per unit of committed capacity. We further assume $q_{\mathrm{t}}=80$ and $q_{\mathrm{c}}=100$ in this paper.

We assume that the subscribers in the local market evaluate ISP's service performance according to the weighted residual bandwidth of ISP's inter-ISP links as follows:

$P_{i}=\frac{t_{i}^{\mathrm{r}} \times\left(c_{i}^{\mathrm{r}}-t_{i}^{\mathrm{r}}\right)+t_{i}^{\mathrm{p}} \times\left(c_{i}^{\mathrm{p}}-t_{i}^{\mathrm{p}}\right)}{t_{i}^{\mathrm{r}}+t_{i}^{\mathrm{p}}}$.

We also assume that the caching function $r(\alpha)$ takes the form of

$r(\alpha)=\gamma_{1}+\gamma_{2} \alpha$

in our case studies. $\gamma_{1}$ and $\gamma_{2}$ vary in different traffic patterns, and new caching technologies or new overlay networks can change their values. Let us take early P2P systems as an example. In early P2P systems, trackers randomly return a list of neighbors to each peer. As a result, 
we can assume all subscribers in one P2P system are homogenous, e.g. they make the same amount of contribution to the system. So the caching function for traffic generated by this kind of P2P systems is $r\left(\alpha_{i}\right)=\frac{\alpha_{i}}{1+\alpha_{0}}$, where $\alpha_{o}$ is the ratio of peers in the remote Internet to peers in the local market. This is equivalent to our model with $\gamma_{1}=0$ and $\gamma_{2}=\frac{1}{1+\alpha_{0}}$.

For any traffic pattern, the caching effect should always be non-negative and should increase with the population size. It implies that $\gamma_{1} \geqslant 0$ and $\gamma_{2} \geqslant 0$. In addition, the marginal cost of a new coming subscriber should be positive in a local ISP, which means that the inter-ISP traffic intensity should increase with the ISP's population size. So we have $\frac{\partial(1-r(\alpha)) \alpha}{\partial \alpha} \geqslant 0$, which is equivalent to $\gamma_{1}+2 \gamma_{2} \leqslant 1$.

ISPs can determine $r(\alpha)$ for the mixed traffic in their market through various measurement technologies, and then apply our game theoretic framework to study provisioning and peering strategies in their market. Researchers can propose reasonable models for any inter-ISP traffic pattern generated by individual popular system. Our game theoretic framework can be easily applied to study the economic implications of the popular system on ISPs' business.

In this section, we fix $\mathrm{ISP}_{2}$ 's private capacity as $c_{2}^{\mathrm{r}}=0.6$ and conduct numerical studies to help ISP $_{1}$ understand the market and make proper decisions.

As we stated at the end of Section 4, Eqs. (20) and (21) are fixed point problems, which reflect the fact that application layer routing might be load-dependent. As a result, we cannot give the closed-form solution of $t_{i}^{\mathrm{r}}$. Therefore $\Phi_{1}\left(\alpha_{1}\right)$ and $\Phi_{2}\left(\alpha_{1}\right)$ in Eq. (7) cannot be presented symbolically. In this work, we solve the problems (20) and (21) numerically using Matlab. For one local market with a particular provisioning setting $c_{i}^{\mathrm{r}}$ and $c_{i}^{\mathrm{p}}$, we calculate $t_{i}^{\mathrm{r}}$ and $t_{i}^{\mathrm{p}}$ numerically for some values of market share distribution $\alpha_{i}$ using an intelligent trial-and-error method. Note that here $\alpha_{i}$ may not be the Nash Equilibrium. If we calculate enough number of pairs $\left(t_{i}^{\mathrm{r}}, \alpha_{i}\right)$, we can derive $\left.\frac{\partial t_{i}^{\mathrm{r}}}{\partial \alpha_{i}}\right|_{\alpha_{i}}$ for any $\alpha_{i}$ approximately. Then we have Eq. (7) in a numerical form. Based on that, we can solve the peering game in Eq. (6) according to Theorem 1 .

\subsection{Provisioning strategy: web traffic}

In this case study, we focus on web traffic, whose routing is controlled by ISPs using BGP. We assume the dominant traffic in current market is web traffic with $\gamma_{1}=0$ and $\gamma_{2}=0.1$. In addition, there will be a new technology that can improve the locality (caching effect) of those web traffic and then $\gamma_{1}=0.05$ and $\gamma_{2}=0.2$.

$\mathrm{ISP}_{1}$ needs to decide two things, e.g. how much capacity it needs to buy and whether to peer with $\mathrm{ISP}_{2}$ or not. The Nash Equilibria under the two traffic patterns in the context of different provisioning and peering strategies are plotted in Fig. 4. Note that each point in the figures shows the Nash Equilibrium of one game, which is defined by a particular provisioning strategy $\left(c_{1}^{\mathrm{r}}\right)$ and a particular peering strategy under the traffic pattern with a particular caching function.
In this market, ISP $_{1}$ can induce more subscribers by increasing the capacity of its private links. After $c_{1}^{r}$ increases, ISP $_{1}$ can charge its subscribers more, while $\mathrm{ISP}_{2}$ has to lower its service price in order to maximize its profit. In this particular market, ISP ${ }_{1}$ 's profit increases after ISP buys more private capacity, while $\mathrm{ISP}_{2}$ 's profit decreases because its price decreases.

Without peering, the market share of the larger ISP (with more private capacity) would increase after the new technology is deployed (such as the deployment of CDNs). It implies that the larger ISP can benefit more from the new technology, which is consistent with economy of scale. The new technology improves the caching effect, so the marginal cost of one subscriber is lowered, which means the competition for subscribers between two ISPs would be fiercer than before. Both ISPs lower its service price to induce subscribers, therefore subscribers also benefit from the technology. However, both ISPs' profits decrease due to the fiercer competition.

Peering is always bad for the ISP with more private capacity when only market share is concerned. This results in that large ISPs do not want to peer with small ISPs. However, both ISPs can charge their subscribers more, thus make more profit after peering. This might explain why ISPs with comparable size would like to peer with each other.

Market share is less sensitive to the provisioning of two ISPs' private links after peering. Peering even reverses the influence of the new technology. After peering, the appearance of the new technology would increase the market share of the small ISP. Peering combines two ISP networks into one single network, thus two ISPs enjoy the same economy of scale. In addition, subscribers even need to pay more after the deployment of the new technology with peering, while without peering the Internet access cost would be lowered after the deployment of the new technology.

\subsection{Provisioning strategy: overlay traffic}

In the second case study we focus on P2P overlay traffic. We assume that the performance sensitivity function $G(\cdot)$ for P2P overlay traffic takes the following form:

$$
G(\delta)= \begin{cases}\frac{1}{2}\left(\frac{\delta}{c_{\mathrm{m}}}\right)^{2 h_{\mathrm{m}} c_{\mathrm{m}}} & \text { if } \delta<c_{\mathrm{m}}, \\ 1-\frac{1}{2}\left(\frac{c_{\mathrm{m}}}{\delta}\right)^{2 h_{\mathrm{m}} c_{\mathrm{m}}} & \text { if } \delta \geqslant c_{\mathrm{m}},\end{cases}
$$

where $h_{\mathrm{m}} c_{\mathrm{m}}>1 / 2$.

$G(\delta)$ is to measure the response of overlay routing to the link performance evaluated by residual capacity of the link. We define the performance sensitivity function in this way because the above function satisfies the following features.

The performance factor approaches zero as the residual capacity of the link approaches zero, i.e. $\lim _{\delta \rightarrow 0} G(\delta)=0$. The traffic on a link with large residual capacity would not be throttled by the link performance, i.e. $\lim _{\delta \rightarrow \infty} G(\delta)=1$. The throttling effect is diminishing as the residual capacity increases, i.e. $G^{\prime}(\delta)>0$.

$h_{\mathrm{m}}$ and $c_{\mathrm{m}}$ are parameters which can be tuned to model traffic patterns generated by different overlays. We believe that $c_{\mathrm{m}}=G^{-1}(1 / 2)$ is also related to the average capacity of 

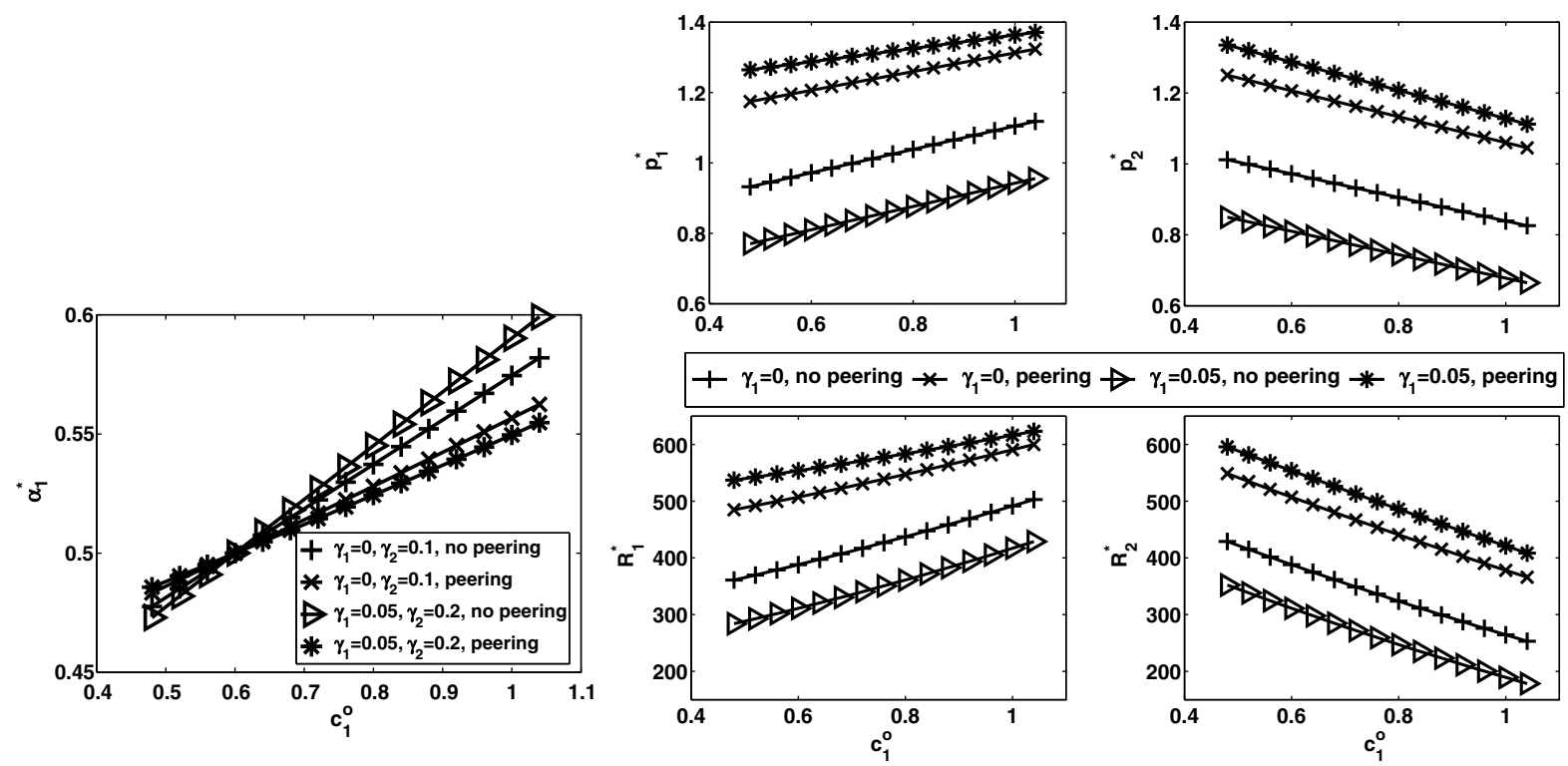

Fig. 4. $\alpha_{1}^{*}, p_{i}^{*}$ and $\mathscr{R}_{i}^{*}$ in different scenarios (web traffic).

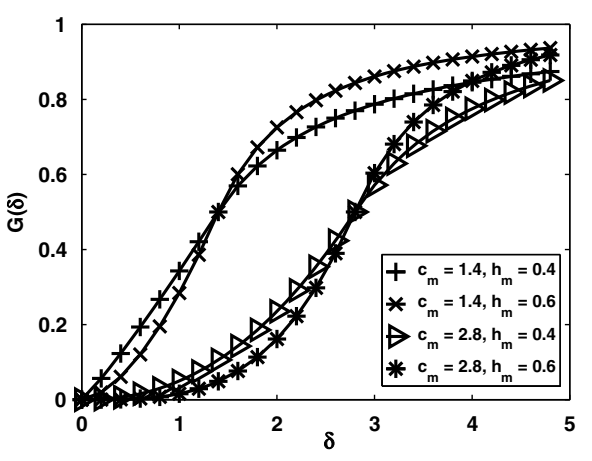

Fig. 5. Performance sensitivity function $G(\delta)$.

intra-ISP links. $h_{\mathrm{m}}$ reflects the sensitivity of the overlay routing to link performance when $\delta$ is around $c_{\mathrm{m}}$, and greater $h_{\mathrm{m}}$ implies more sensitive overlay routing. Fig. 5 is an illustration of $G(\delta)$ with different parameters.

In this case study, the first traffic pattern under study is with $\gamma_{1}=0, \gamma_{2}=0.1, c_{\mathrm{m}}=1.4$ and $h_{\mathrm{m}}=0.4$. People may propose various ways to improve locality and routing sensitivity of overlay traffic. Our second traffic pattern is used to model this trend by setting $\gamma_{1}=0.1, \gamma_{2}=0.2, c_{\mathrm{m}}=1.4$ and $h_{\mathrm{m}}=0.6$. As far as we know, currently there is no research result on the relation between link performance and application layer routing choice (i.e. performance sensitivity function). The only reference we can find to help us determine $G(\delta)$ is a few measurement results mentioned in [2].

Similar as the first case study, we solve all the games with different provisioning profiles, peering strategies and different traffic patterns. The resulting Nash Equilibria of these games are plotted in Fig. 6 .
When two ISPs do not peer with each other, increasing private capacity results in more subscriber share. After peering, however, increasing the provisioning reduces its own market share and increases its competitor's profit. It might be caused by overlay routing, which renders more difficulties for ISPs to achieve their particular goals. ISP ${ }_{1}$ increases $c_{1}^{r}$, and it makes that more peers in $\mathrm{ISP}_{1}$ retrieve information from outside peers instead of from peers in the local market due to performance-sensitive routing [2]. Therefore, ISP ${ }_{1}$ 's inter-ISP intensity increases, which may partly counteract the performance improvement from the increased provisioning. At the same time, $\mathrm{ISP}_{1}$ needs to pay more because of the increased capacity and increased inter-ISP traffic intensity. As a result, ISP $_{1}$ has to increase the price although its performance improvement is limited, which may drive away its subscribers. Comparing with the market with only web traffic, it is more difficult for two ISPs to make a free peering agreement due to application layer routing.

Fig. 6 show that it might be a good strategy for one ISP to reduce its private link provisioning under the peering scenario. The ISP's market share does not decrease and its profit increases. This may explain why some ISPs begin to use bandwidth throttling to contain inter-ISP P2P traffic intensity. Obviously, with peering relationship, the traffic pattern under study discourages network growth and service improvement under current Internet architecture and business model.

Before peering, new technologies that improve the locality and the performance sensitivity of overlay applications would also make the competition between two ISPs fiercer. ISPs reduce their price to induce more subscribers because subscribers themselves are serving as information providers and thus become more valuable for ISPs. After peering, both ISPs can make use of all peers in the whole 

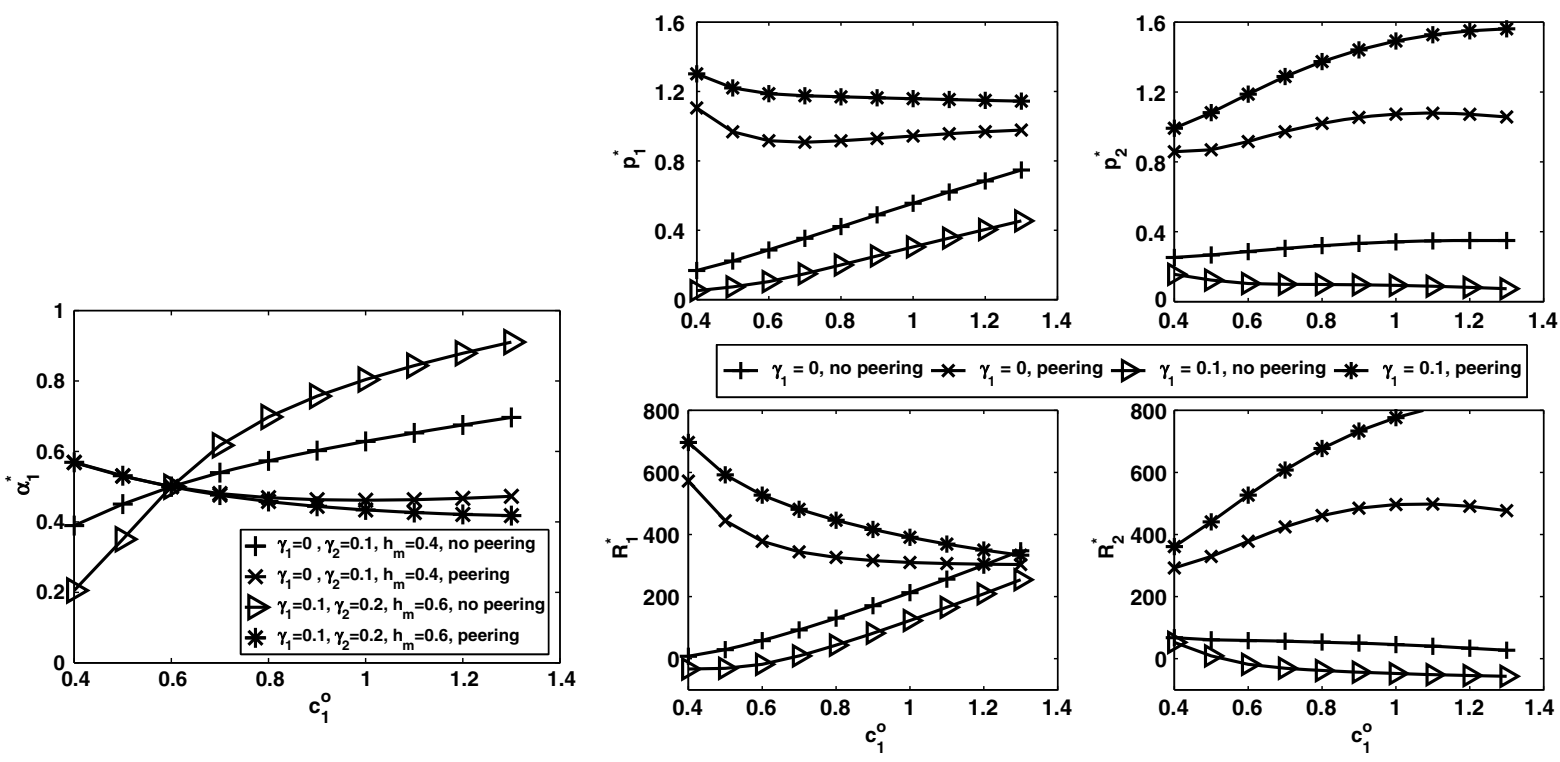

Fig. 6. $\alpha_{1}^{*}, p_{i}^{*}$ and $\mathscr{R}_{i}^{*}$ in different scenarios (overlay traffic).

market, so it may not be necessary for them to reduce the service price to attract subscribers.

In terms of market share, peering is always bad for the ISP with larger capacity. The negative influence is more apparent in a market with overlay traffic than a market with web traffic. But after peering two ISPs can increase their prices at the same time, thus make more profit. To some extent, we can say that two ISPs are partly cooperating with each other under the peering scenario.

\subsection{Case study: peering strategy}

Now let us look into the peering strategy. Peering strategy is more than deciding peering or not. ISPs can control the peering capacity between them to achieve their individual goals. In some networks, peering capacity has significant influence on ISPs' business.

In this case study, the local market is the same as before. We further assume ISP ${ }_{1}$ 's private capacity is also fixed and $c_{1}^{r}=1$. We will look at the influence of peering capacity in the context of three traffic patterns. These three traffic patterns are with the same caching function where $\gamma_{1}=0$ and $\gamma_{2}=0.1$. The first traffic pattern is web traffic. The other two traffic patterns are overlay traffic patterns with $c_{\mathrm{m}}=1.4$ and $h_{\mathrm{m}}=0.4$ or 0.6 . The Nash Equilibria $\left(\alpha_{1}^{*}, p_{i}^{*}, \mathscr{R}_{i}^{*}\right)$ of peering games under different peering capacities are plotted in Fig. 7.

For web traffic, the routing is determined by ISPs, and only local traffic can go through the peering link. So the traffic intensity on the peering link is always below a certain value. $\alpha_{1}^{*}$ would not be reduced a lot by increasing peering capacity. However, overlay traffic is not under the control of the peering agreements. Overlay applications can continue moving traffic to the path with good performance, so increasing peering capacity exasperates the free-riding of the ISP with worse performance. It may reduce the market share of the larger ISP $\left(\alpha_{1}^{*}\right)$ a lot when overlay traffic dominates. In addition, the negative influence would be larger if the overlay traffic is more performance-sensitive. Therefore, with overlay traffic, ISPs should be very careful when making the peering decisions.

We also notice that ISPs' prices keep increasing as two ISPs increase their peering capacity. It reveals that two ISPS form a tighter coalition and they have more bargaining power to subscribers after their peering capacity increases, although the peering benefit distribution is not fair, which can be seen from the change of the market share distribution shown in Fig. 7.

\subsection{Discussion on subscribers' choices}

In the above analysis, two ISPs are able to provide satisfactory service for all subscribers in this market (or Internet access service is necessary for subscribers in this market), so what the subscribers need to decide is which ISP to subscribe. This market can be thought as saturated, where all potential subscribers have joined the Internet. These subscribers always choose the ISP with high service utility, thus the utility of subscribers in two ISPs evaluate to the same value when the market becomes stable. In this model, two ISPs are competing with each other, and both of them have incentives to lower the price to attract subscribers from the other ISP. As a result, subscribers enjoy reasonable prices.

We can allow that some of $n$ potential subscribers do not subscribe to any ISP to model an expanding market. In this market two ISPs are not able to provide satisfactory service for all potential subscribers. One subscriber always chooses the ISP with high service utility, and the service utility must be non-negative. Otherwise, the subscriber does not subscribe the Internet access service. With this assumption, we still have the following equation for the Nash Equilibrium:

$\mathscr{U}_{1}\left(p_{1}^{*}, \alpha_{1}^{*}\right)=\mathscr{U}_{2}\left(p_{2}^{*}, \alpha_{2}^{*}\right)$. 

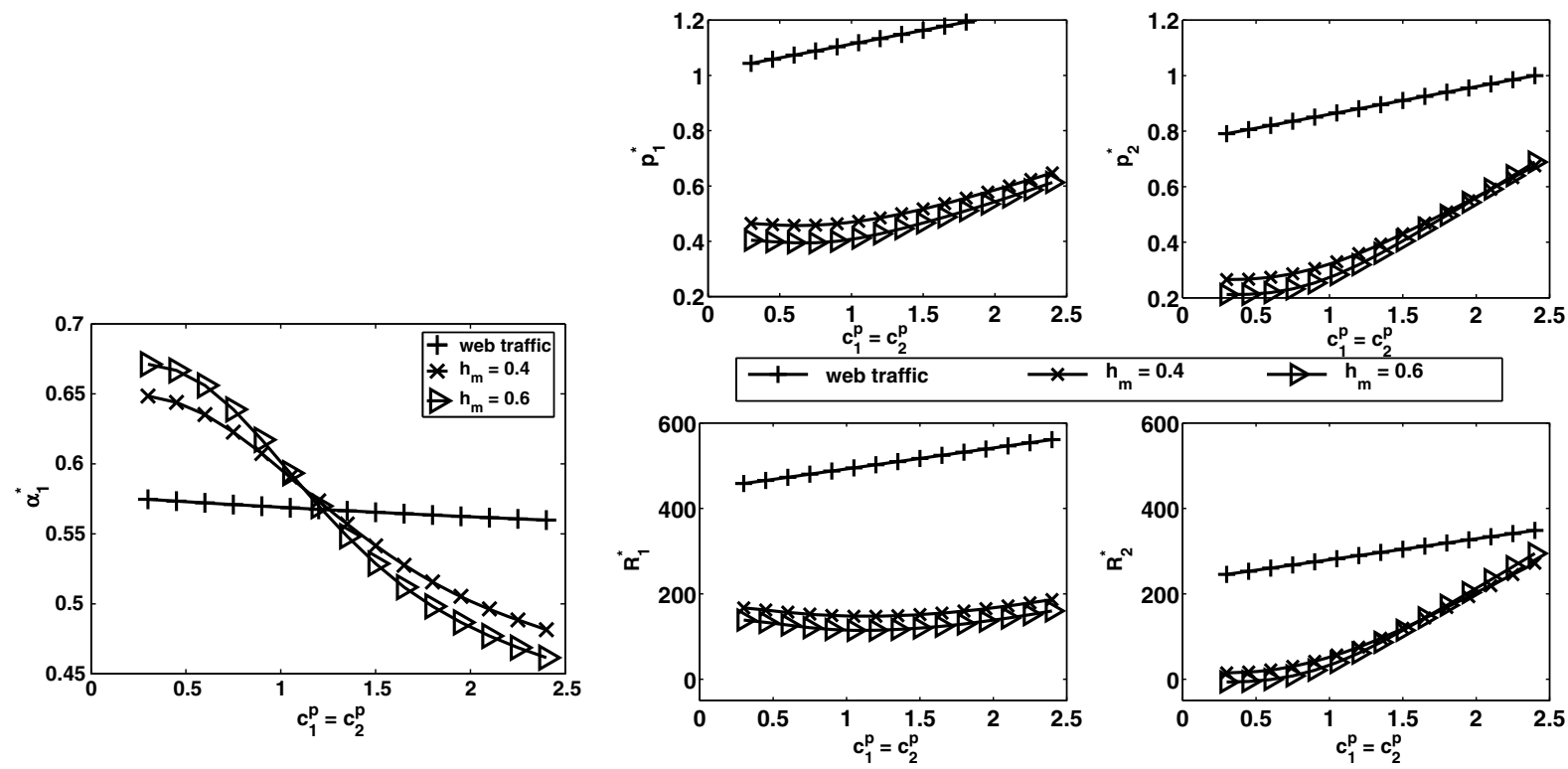

Fig. 7. $\alpha_{1}^{*}, p_{i}^{*}$ and $\mathscr{R}_{i}^{*}$ under different peering capacities.
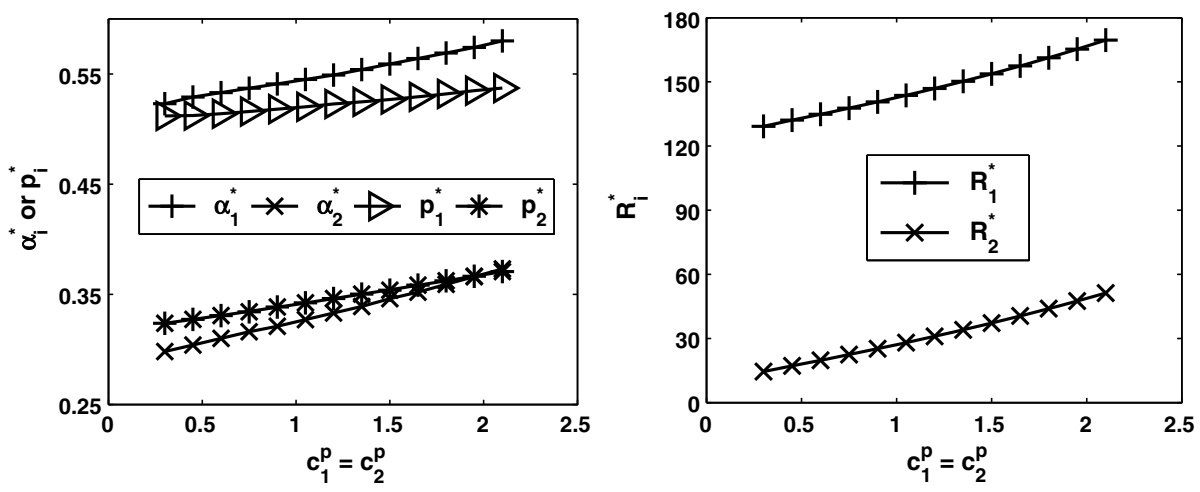

Fig. 8. Some subscribers do not choose Internet access service.

There is one more condition for the Nash Equilibrium as follows:

$\mathscr{U}_{1}\left(p_{1}^{*}, \alpha_{1}^{*}\right) \geqslant 0, \quad \mathscr{U}_{2}\left(p_{2}^{*}, \alpha_{2}^{*}\right) \geqslant 0$.

Let us assume that some users do not choose any ISP when the market becomes stable, i.e. $\alpha_{1}^{*}+\alpha_{2}^{*}<1$ at the Nash Equilibrium point. Obviously we have

$\mathscr{U}_{1}\left(p_{1}^{*}, \alpha_{1}^{*}\right)=0, \quad \mathscr{U}_{2}\left(p_{2}^{*}, \alpha_{2}^{*}\right)=0$.

Otherwise subscribers would choose the ISP with positive utility. In this case, two ISPs are not competing with each other directly since there are "free" potential subscribers which do not belong to any ISP.

We conduct the numerical study under the new assumption for the web traffic in the third case study. ${ }^{1}$ The result is shown in Fig. 8.

We have two observations from Fig. 8. First, the two ISPs' prices are lower than the result in Fig. 7. In order to

\footnotetext{
${ }^{1}$ We ignore the two overlay traffic patterns since two ISPs provide positive utility for all subscribers in the market at Nash Equilibria points under those two overlay traffic patterns.
}

avoid some subscribers leaving the Internet, both ISPs lower their prices to attract potential subscribers. However, the same as in a saturated market, the two ISPs' prices keep increasing as they increase the peering capacity. Second, the market shares of both ISPs are increasing with the peering capacity, but the the market share of the smaller ISP increases more quickly than the larger ISP, which shows that the smaller ISP benefits more from the peering relationship than the larger ISP.

This case study is for an expanding market, where two ISPs cannot provide satisfactory service for all potential subscribers; while the first three case studies can be viewed as for a saturated market, where all subscribers have joined the Internet and two ISPs are competing with each other directly.

\section{Conclusion}

In this paper, we study a local market with two ISPs competing with each other for market share of subscribers. We formulate the interaction among ISPs and subscribers 
in this market as a two-stage game and study the influence of different traffic patterns on the Nash Equilibrium of the market. The goal of this paper is to establish an abstract and quantitative framework that brings out the important factors that affect ISP peering. ISPs can also use this methodology to build a decision support system to study its peering and provisioning strategies.

We provide a general inter-ISP traffic model to define traffic pattern based on caching function and performance sensitivity function. Under this framework, we focus on two traffic pattern families. One is for the traditional web traffic, whose routing is determined by ISPs. The other is for overlay traffic, which represents the traffic whose routing is reactive to the network performance. We conduct numerical studies to understand the influence of traffic patterns on the peering game in the market. The result reveals some important observations on ISPs' business. First, economy of scale plays an important role in ISPs peering game. Second, in certain scenarios, application layer routing might discourage network growth and service improvement because current network architecture and business model do not take this kind of traffic into consideration. Third, even when P2P overlay traffic dominates, local peering is still beneficial for local ISPs as a total although the peering benefit distribution may not be fair.

Actually, free-riding, which refers to the situation that ISPs provide unintended service for other ISPs, can also occur in other selective-transit scenarios. For example, a multi-homing ISP may unknowingly provide transit service for its providers, due to P2P or other overlay applications. In this paper, we focus our attention to the case of free-riding when local ISPs are engaged in a settlement-free peering agreement. This situation drew our attention because it really occurs and has become a concern by a local ISP we consult with.

Our dynamic market analysis involves assumptions on transmission cost function between ISPs, subscribers' utility function to choose their own provider and inter-ISP traffic model (Web and overlay). The transmission cost is dependent on link capacity and traffic intensity between two ISPs. In our utility function, subscribers take price and performance of each ISP into consideration when they make their decision. The inter-ISP traffic intensity is related to population distribution, ISPs' caching efficiency and routing sensitivity to link provisioning. Although the particular form of these functions in this paper should be further validated, we believe the above factors are critical and essential for local market analysis.

In this paper, the caching function together with performance sensitivity function is provided as a general framework to model a family of different inter-ISP traffic patterns. The inter-ISP traffic pattern is a significant factor that influences ISPs' economic behavior. However, as far as we know, currently there is no research result on describing the internal and external traffic of one ISP based on its population size and its network provisioning. We are now further studying this issue through measurements and simulations to improve and validate our traffic model. However, ISPs can conduct measurement on their own network to determine their traffic pattern, and then apply our game-theoretic framework during their own economic analysis.

The analysis in this paper does not take into consideration any peering constraint between ISPs. In the real world, two ISPs forming bilateral charge-free peering agreements require that traffic flowing between them should be roughly equal. Otherwise they would not agree to freely peer. Under this situation, the extreme cases would not occur. However, from our analysis we can see that this constraint is more important than before for large ISPs after P2P overlays appear (see Fig. 7). Moreover, it also explains why some large ISPs disconnected from public multilateral peering points, e.g. HKIX.

The network architecture, along with the traffic engineering policies that ISPs use, are based on certain assumptions about how their customers and traffic behave. Overlay networks could call into question some of these assumptions, thus rendering it more difficult for ISPs to achieve their goals. We believe that this study is helpful for ISPs to have better knowledge about various traffic patterns to cope with the different technical issues and business issues, and the insight of this work may lead to further examination of the Internet architecture and inter-domain routing mechanisms. Therefore, we believe this research can potentially have high practical as well as theoretical significance.

\section{References}

[1] S. Banerjee, C. Kommareddy, B. Bhattacharjee, Efficient peer location on the internet, Computer Networks 45 (1) (2004) 5-17.

[2] R. Bindal, P. Cao, W. Chan, J. Medved, G. Suwala, T. Bates, A. Zhang, Improving traffic locality in bittorrent via biased neighbor selection, in: 26th IEEE International Conference on Distributed Computing Systems, 2006, ICDCS, 2006, pp. 66-77.

[3] H. Chang, S. Jamin, M. Mao, W. Willinger, An empirical approach to modeling inter-as traffic matrices, in: IMC'05, 2005 Internet Measurement Conference, Berkley, CA, USA, 2005.

[4] B.-G. Chun, K. Chaudhuri, H. Wee, M. Barreno, C.H. Papadimitriou, J. Kubiatowicz, Selfish caching in distributed systems: a gametheoretic analysis, in: PODC'04: Proceedings of the 23rd Annual ACM Symposium on Principles of Distributed Computing, ACM Press, New York, NY, USA, 2004, pp. 21-30.

[5] M. Fukushima, J. Pang, Some feasibility issues in mathematical programs with equilibrium constraints, SIAM Journal of Optimization 8 (1998) 673-681.

[6] J. Glasner, P2p fuels global bandwidth binge, Wired News (2005). April.

[7] G. Huston, Interconnection, peering and settlements. inet, 1999. available at <http://www.isoc.org/inet99/proceedings/1e/1e_1. htm>.

[8] R. Johari, J. Tsitsiklis, Routing and Peering in a Competitive Internet, MIT Tech, 2003.

[9] R. Keralapura, N. Taft, C.N. Chuah, G. Iannaconne, Can ISPs take the heat from overlay networks? in: ACM HotNets, III, November 2004.

[10] S. Leyffer, T. Munson, Solving multi-leader-follower games, Preprint ANL/MCS-P1243-0405, 2005

[11] W.B. Norton, Internet service providers and peering, Technical report, Equinix, 2001.

[12] W.B. Norton, A business case for ISP peering, Technical report, Equinix, 2002.

[13] W.B. Norton, The evolution of the us internet peering ecosystem, Technical report, Equinix, 2003.

[14] J.V. Outrata, Optimality conditions for a class of mathematical programs with equilibrium constraints, Mathematics of Operations Research 24 (3) (1999) 627-644.

[15] J.-S. Pang, M. Fukushima, Quasi-variational inequalities, generalized Nash equilibria and multi-leader-follower games, Computational Management Science 2 (1) (2005) 21-56. January. 
[16] S. Seetharaman, M. Ammar, On the interaction between dynamic routing in the native and overlay layers, in: INFOCOM 2006 , Barcelona, Spain, April 2006.

[17] S. Shakkottai, R. Srikant, Economics of network pricing with multiple ISPs, in: Proceedings IEEE 24th Annual Joint Conference of the IEEE Computer and Communications Societies, INFOCOM 2005, vol. 1, 2005, pp. 184-194.

[18] K. Tutschku, P. Tran-Gia, Traffic characteristics and performance evaluation of peer-to-peer systems, in: Peer-to-Peer Systems and Applications, Springer, 2005, pp. 383-397.

[19] H. Wang, D.M. Chiu, J.C. Lui, Modeling the peering and routing tussle between ISPS and p2p applications, in: Proceedings of the 14th IEEE International Workshop on Quality of Service (IWQoS 2006), New Haven, USA, June 2006, pp. 51-59.

[20] M.B. Weiss, S.J. Shin, Internet interconnection economic model and its analysis: Peering and settlement, Netnomics 6 (1) (2004) 43-57.

[21] Y. Zhang, M. Roughan, N. Duffield, A. Greenberg, Fast accurate computation of large-scale ip traffic matrices from link loads, in: SIGMETRICS'03, Proceedings of the 2003 ACM SIGMETRICS International Conference on Measurement and Modeling of Computer Systems, ACM Press, New York, NY, USA, 2003, pp. 206217.

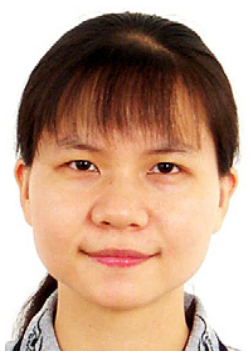

Jessie Hui Wang received her Ph.D. degree from the Department of Information Engineering at the Chinese University of Hong Kong. She is now an assistant research professor in Tsinghua University. Her research interests include traffic engineering, routing protocols and economic analysis of data networks.

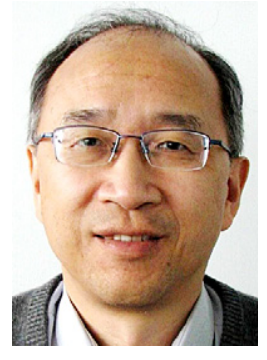

Dah Ming Chiu received the B.Sc degree from Imperial College London and the Ph.D. degree from Harvard University in 1975 and 1980. After twenty years in industry (Bell Labs, DEC and Sun), he is now a professor in the Department of Information Engineering in CUHK. His research interest includes Internet, wireless networks and P2P networking. He is an editor for IEEE/ACM Transactions on Networking, and TPC member for various IEEE conferences including Infocom, ICNP and IWQoS.

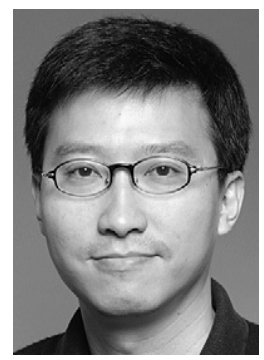

John C.S. Lui received his Ph.D. in Computer Science from UCLA. He later joined the Department of Computer Science and Engineering at the Chinese University of Hong Kong. His research interests include theoretic/ applied topics in data networks, distributed multimedia systems, network security and mathematical optimization and performance evaluation theory. John was the TPC co-chair of ACM Sigmetrics 2005 and the general cochair of the International Conference on Network Protocols 2006. His personal interests include films and general reading. 